\title{
Finsler Level Set Segmentation for Imagery in Oriented Domains
}

\author{
Vandana Mohan, John Melonakos and Allen Tannenbaum \\ Electrical and Computer Engineering, \\ Georgia Institute of Technology, Atlanta GA, USA \\ Marc Niethammer \\ Surgical Planning Laboratory, \\ Brigham and Women's Hospital, Boston MA, USA \\ Marek Kubicki \\ Laboratory of Mathematics in Imaging, \\ Brigham and Women's Hospital, Boston MA, USA \\ vandana.mohan@gatech.edu
}

\begin{abstract}
In this paper, we present a novel directional level set segmentation framework employing the theory of Finsler active contours. The framework provides a natural way to perform segmentation of image data in oriented domains. We share examples of this technique on diffusion-weighted magnetic resonance imagery (DW-MRI) for the segmentation of neural fiber bundles and we show examples of texture based segmentation using structure tensors. We also demonstrate that for some applications higher accuracy is achieved by the proposed framework than by level set methods that employ Riemannian metrics. This gain is attributed to the relaxation of the tensor model constraint which is imposed upon the metric in the Riemannian case.
\end{abstract}

\section{Introduction}

Image segmentation is the problem of separating a given image into areas of interest, based on image-derived features. These features could include intensity and edge information, regional statistics, etc. In many scenarios, it is beneficial to transform the image into a space where the features of interest can be better discriminated. One such instance is when intensity information is insufficient to segment an image. In this case, it is desirable to use additional features to help discriminate the object of interest from the background (such as using structure tensors to capture textural information). Interesting approaches in this vein include recent work on the application of structure tensors for image segmentation [2]. The structure tensor is a feature that essentially converts the given isotropic image data into an oriented domain. In this work, we present a method which is able to segment features of interest in oriented domains of arbitrary dimension via the level set method. In addition to the structure tensor example, there are other naturally occurring instances of 
imagery in oriented domains - for example, diffusion-weighted magnetic resonance imagery (DW-MRI). In this work, we build upon our previous work based on Finsler active contours [3]. Specifically, we report the first formulation of this Finsler level set method in arbitrary dimension and the first application of the framework to a variety of oriented domains minimizing novel energy-based direction-dependent functionals.

The segmentation problem in oriented domains is interesting because the image data is a function of not only position but also direction. Since the segmentation of DW-MRI data is inherently a directional problem, and texture segmentation can be recast as the segmentation of an oriented image, it is expected that a segmentation framework which naturally incorporates directionality - like the Finsler active contour framework - can be applied for both in fairly similar ways.

There has been a great deal of research in the direction of finding and characterizing neural connections between brain structures in diffusion-weighted magnetic resonance imagery. The cingulum bundle (CB) is a structure in the brain that has recently become the subject of interest as an anatomical structure which might display quantifiable differences between schizophrenic and normal control populations and thus aid towards the diagnosis of this disorder. The cingulum bundle is a fiber bundle and thus made up of several individual fibers, mostly disjoint and potentially intersecting at points, all roughly similarly aligned. Thus the fiber bundle clearly possesses distinct directionality from the surrounding areas of the brain, which could be either intersecting fibers running roughly perpendicular to the $\mathrm{CB}$, or structures with strong but isotropic diffusion profiles like the cerebral spinal fluid (CSF).

It should be noted that segmentation using a Riemannian approach can work with tensor data (and thus handle the structure tensors), however it enforces an elliptic constraint on the data, by the very nature of approximation of these tensors. There are usage scenarios where the data at hand might not be accurately captured by a tensor model. Thus, extracting textural information as a tensor is an example of a situation where generality in the nature of this feature might allow for better representation and thus better segmentation. The Finsler active contour framework presented in this paper offers a natural way to work with such general directional features.

In this paper, we extend the results of [3] by presenting the first formulation and results of a level set implementation of this framework in arbitrary dimension. We have successfully applied this technique to DW-MRI data and tested its application in the case of image segmentation using textural information. We also illustrate the advantage of the framework in its ability to handle directional data that is not expressed (or approximated) to fit a tensor model, and the increase of accuracy of the segmentation obtained as a result.

\subsection{Textural Segmentation}

The problem of texture-based segmentation has been explored extensively in [2] and [5] and the references there-in. The motivation for the application of this framework to textural segmentation is to exploit the directionality of the feature - the structure tensor - being extracted. As opposed to current methods that employ regional statistics on the structure tensor space, this work finds edges in this oriented space. Thus, it can also easily be extended to images of any higher dimension. As shall be discussed subsequently in this paper, the Finsler-based framework removes the need to fit a tensor model to the data, and can thus be expected to better discriminate features of interest. 


\subsection{DW-MRI Segmentation - Identification of the Cingulum Bundle}

The cingulum bundle is a $5-7 \mathrm{~mm}$ in diameter fiber bundle that interconnects all parts of the limbic system. It originates within the white matter of the temporal pole, and runs posterior and superior into the parietal lobe, then turns, forming a "ring-like belt" around the corpus callosum, into the frontal lobe, terminating anterior and inferior to the genu of the corpus callosum in the orbital-frontal cortex [6]. Moreover, the CB consists of long, association fibers that directly connect temporal and frontal lobes, as well as shorter fibers radiating into their own gyri. The $\mathrm{CB}$ also includes most afferent and efferent cortical connections of cingulate cortex, including those of prefrontal, parietal and temporal areas, and the thalamostriatae bundle. In addition, lesion studies document a variety of neurobehavioral deficits resulting from a lesion located in this area, including akinetic mutism, apathy, transient motor aphasia, emotional disturbances, attentional deficits, motor activation, and memory deficits. Because of its involvement in executive control and emotional processing, the $\mathrm{CB}$ has been investigated in several clinical populations, including depression and schizophrenia. Previous studies, using DTI, in schizophrenia, demonstrated decrease of FA in anterior part of the cingulum bundle [1,8], at the same time pointing to the technical limitations restricting these investigations from following the entire fiber tract.

Given the directional nature of the cingulum bundle, it is of interest to test the efficacy of the proposed framework in automatically segmenting this structure from a given 3D brain volume.

\section{Background}

\subsection{Motivation}

For image data in oriented domains, the boundaries of objects of interest extremize energy functionals that depend not only on position but also on direction. This is the fundamental motivation behind the Finsler active contour framework since it attempts to extremize an energy of exactly this form. Prior work has implemented the dynamic programming based approach to this framework. The work documented in this paper documents the first level set implementation of this framework in arbitrary dimension and the results obtained with this implementation for 2D and 3D images. This implementation has the natural advantages of being able to handle topological changes, and being able to trivially extend our implementation to arbitrary dimension. Also, it has the advantage that it can accommodate a variety of cost functions since it accepts the cost function as a scalar field and thus the actual implementation requires no alterations from the basic setup.

\subsection{Theory and math}

Let $\Psi(p, d)$ denote the energy that we desire to minimize as a function of the position ' $\mathrm{p}$ ' and the direction ' $d$ '. The expression for the evolution of the hypersurface is obtained as,

$$
\Sigma_{t}=-\left\{\nabla_{p} \Psi . N+\operatorname{Tr}\left(\nabla_{d d} \Psi\right)+\Psi . \operatorname{Tr}(d N)\right\} N
$$

where $\Sigma$ denotes the hypersurface, $\Psi$ is the local cost, $N$ denotes the unit normal to the hypersurface, $d N$ is the shape operator. The detailed derivation of this expression can be 
found in [4].

Denoting the surface of interest as the zero level set of a signed distance function, we obtain the expression for the evolution of the level set function as,

$$
u_{t}=\nabla_{p} \Psi \cdot \nabla u+\left\{\operatorname{Tr}\left(\nabla_{d d} \Psi\right)+\Psi \cdot \operatorname{Tr}(d N)\right\}\|u\|,
$$

where $u$ is the level set function.

This expression can be separated into the terms that influence diffusion and terms that are hyperbolic in nature. In the numeric implementation of this framework, appropriate differencing methods are employed for the individual terms depending upon which category they belong to.

\section{Implementation}

The implementation of the Finsler active framework documented in this paper is done in arbitrary dimension. The terms from the expression for the hypersurface evolution that are challenging to compute in arbitrary dimension are the shape operator, $d N$, and surface derivatives at each point on the on the hypersurface. In this work, the level set approach of [7] is used to implement this framework and by an appropriate choice of the level set function as a signed distance function, it is possible to obtain simplified expressions for these two terms as discussed in subsequent sections.

\subsection{Level Set Implementation}

The level set formulation implicitly represents the surface of interest as the zero level set of an appropriately defined level set function. This work uses the signed distance function because of its desirable properties with regards to the computation of geometrical quantities of interest in surface evolution. This choice simplifies the computation of the Shape operator, $d N$, and a basis for the tangent space (which is equivalent to the surface derivatives with respect to the arc length parameter $s$ ).

\subsection{Shape operator}

Let $u$ denote the signed distance function (SDF). Then, the normal can be obtained from the gradient of the SDF as,

$$
N=\frac{\nabla u}{\|\nabla u\|} .
$$

Since the shape operator is the derivative of the normal to the surface with respect to arc length, $s$, we can write it as,

$$
\frac{d N}{d s}=\frac{d}{d s} \frac{\nabla u}{\|\nabla u\|} .
$$

This can be simplified to obtain an expression for the shape operator in terms of the SDF as

$$
d N=\frac{1}{\|\nabla u\|}\left(I-N \cdot N^{t}\right) H_{u} .
$$

where $H_{u}$ is the pointwise hessian of the levelset function 'u'. 


\subsection{Computation of surface derivatives}

It can be seen that that with arc length parameterization and the fact that the surface derivatives required in the numeric implementation occur under a trace operator (which is invariant under a change of basis of the matrix argument or a change of order of arguments), we can replace the matrix containing surface derivatives by an arbitrary choice of basis for the tangent space at the point. This basis is trivial to determine if the unit normal to the surface is known. Since for an SDF, the normal can be obtained from the gradient, we can simply express the matrix with the tangent space basis as,

$$
d \Sigma=\left(I-N \cdot N^{t}\right)
$$

where $d \Sigma$ denotes the surface derivatives used in the evolution, and $N$ denotes the unit normal to the surface $\Sigma$.

It can be shown that the eigenvectors of this matrix are in fact the normal itself - corresponding to zero eigenvalue - and the (n-1) tangent directions corresponding to eigenvalue 1 which represent surface derivatives.

The sparse field approach of [9] was chosen to implement the level set formulation of the Finsler active contour framework, in order to achieve fast computations given the large sizes of the directional datasets being used.

\subsection{Cost functions}

The fundamental motivation to all the cost functions explored in this work is to find edges in the directionality of the underlying data. Hence all the cost functions discussed here yield segmentations that are primarily edge-based in nature. A crucial feature of the available data is that the object of interest does not homogeneously display a single direction. There are intermediate areas where (using DW-MRI terminology), the diffusion is possibly isotropic and sometimes zero. Thus, it is important to suitably compute gradients in these images to better capture the directionality inside (and outside) the evolving surface. The cost functions are formulated in such a way that the costs ranges from 0 to 1 , and so that the energy is minimized on the surface of interest. The convergence condition was fairly simple with the algorithm stopping when the average cost on the surface fell below 0.1 and stayed there beyond a certain number of iterations.

\section{Experiments and Results}

The framework was tested via three sets of experiments. The first aimed to compare it with the Riemannian approach on a tensor fit of the data. The second experiment applied the framework to the segmentation of a texture image and the third applied it towards automatically finding the cingulum bundle from DW-MRI imagery.

\subsection{Synthetic data}

One of the advantages of the Finsler active contour framework is the fact that the data does not need to be made to fit a tensor model. This allows greater accuracy in capturing features of interest. This is demonstrated via a comparison between the proposed framework and a Riemannian approach applied to a tensor approximation of the same data set. 
This data set is synthetic with different diffusion profiles within and outside the object of interest which is a simple circle. It is an extreme example since the diffusion profiles within and outside the circle look identical when fit to a tensor model (Table 1, and the Riemannian approach eventually causes the initial surface to just collapse to a point under the influence of curvature, since under the tensor model, the energy landscape is flat and 1 (maximum) everywhere! This experiment though simplified, clearly illustrates the advantage of our framework.

\subsection{Texture Segmentation}

For textural segmentation, the transformation of the available data into an oriented domain is crucial to the effectiveness of the segmentation algorithm. This transformation is dependent upon the nature of the image that we desire to segment. In the images used in this work, it is of interest to separate the directionality of the gradients of the image inside and outside the object. Hence, the transformation that we employ to construct the feature data for this application is to compute the directional gradients of the given image. The gradients are comparable to the diffusion profile for DW-MRI imagery and the directions in which these gradients are computed are analogous to the sampling directions. Figure 2 illustrates this. Figure 3 shows a comparison of the method with the intensity edge-based approach, and clearly demonstrates the advantage we gain by adding directionality into the segmentation problem via the transformation into an oriented domain. It is observed that with a purely intensity edge-based approach, for this image, the segmentation process is not well-behaved and does not converge.

\subsection{DW-MRI data}

The object of interest in the available DW-MRI imagery is the cingulum bundle. We can deduce the equivalence between such a dataset and the image on which the textural segmentation has been demonstrated. The 'stripes' in the textural image are in a sense equivalent to the fibers within the cingulum bundle that we desire to encapsulate within a single surface. The same cost function was in fact applied to both these data sets with no difference other than the dimension of the data itself. The initial level set for this application was arrived at by creating the surface of smallest volume that fully contains the anchor tract that is obtained out of the Finsler tractography module discussed in [3].

In DW-MRI data, the task of segmenting the cingulum bundle is equivalent to find disjoint fibers in the available brain volume that are similarly aligned. In the absence of ground truth for the cingulum bundle in the available data, we use the availability of simple eigenvalue analysis based approximations to the cingulum bundle along with visual verification to validate the performance of the algorithm on the DW-MRI data. Based on these, the CB extracted is found to be satisfactory as shown in figure 4.

\section{Conclusions and Future Work}

In this work, we have introduced the Finsler level set framework which is a novel way to perform segmentation of imagery in oriented domains. We demonstrate the advantage of the framework over Riemannian approaches that impose a tensor model on the data. We have also shown the application of this framework in segmenting the cingulum bundle 
Table 1: Diffusion profiles in the synthetic image. Results of comparison in Figure 1

\begin{tabular}{|c||c|c|}
\hline \hline & Diffusion profile inside & Diffusion profile outside \\
\hline Original data & & \\
Tensor model approximation & &
\end{tabular}

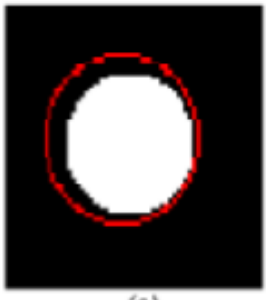

(a)

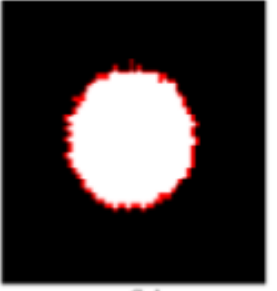

(b)

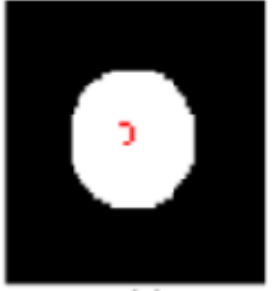

(c)

Figure 1: Results on synthetic data comparing Finsler-based framework to Riemannian approach using tensors. (a) shows the initial level set. (b) shows the result obtained using the Finsler level set framework, and (c) shows the result of applying Riemannian level sets on a tensor fit to the data. (Diffusion profiles are provided in Table 1)

from DW-MRI imagery and a proof-of-concept of its utilization in texture segmentation, while simultaneously drawing the parallels between the two cases that allow us to use similar approaches. The algorithm is found to perform well in both these cases.

The framework gives us a method to automatically segment the cingulum bundle from the DW-MRI volume. The results have been validated by comparison with eigen value based thresholding and visual inspection. Further validation will be explored by incorporating the algorithm into a system that can automatically extract the $\mathrm{CB}$ and perform statistical analysis on the CB towards differentiating normal controls from schizophrenic controls. Also, the texture segmentation examples shown in this paper have been chosen because of their similarity to the nature of the DW-MRI data in the sense of separating directionality inside and outside the object, and to demonstrate the advantage of adding directionality to the image segmentation framework. Future work will attempt to apply this framework to more texture images. Also, since the costs employed in this work are 


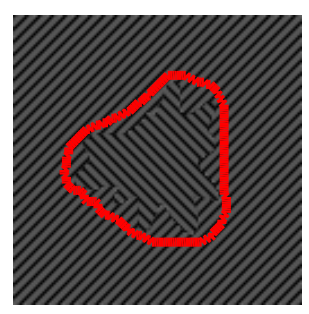

(a)

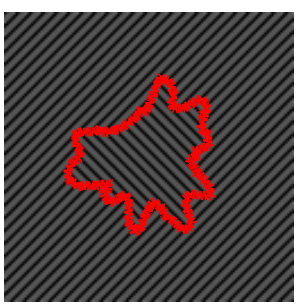

(b)

Figure 2: Result on texture image. (a) shows the initial levelset and (b) shows the (smoothed) result from the Finsler levelset framework

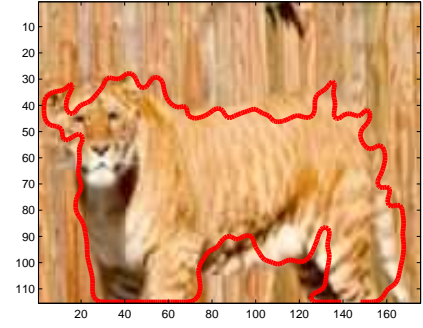

(a)

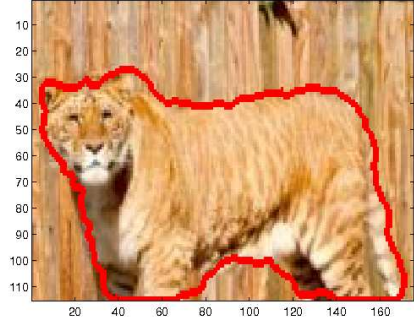

(b)

Figure 3: Comparison between (a) intensity edge-based approach and (b) directional edge-based (proposed) approach for same initial level set.

purely edge-based and hence we face natural limitations on the placement of the initial levelset, it is also of great interest to explore costs of a more regional nature and evaluate their effectiveness in texture segmentation as well as on the DW-MRI data.

\section{References}

[1] M. Kubicki, C.F. Westin, P.G. Nestor, C.G. Wible, M. Frumin, S.E. Maier, R. Kikinis, F.A. Jolesz, R.W. McCarley, and M.E. Shenton. Cingulate fasciculus integrity disruption in schizophrenia: a magnetic resonance diffusion tensor imaging study. Biological Psychiatry, 54(11):1171-1180, 2003.

[2] J. Malcolm, Y. Rathi, and A. Tannenbaum. A graph cut approach to image segmentation in tensor space. In Workshop on Component Analysis Methods (CVPR), 2007.

[3] J. Melonakos, E. Pichon, S. Angenent, and A. Tannenbaum. Finsler Active Contours. To appear in IEEE Trans. PAMI, 2007. 


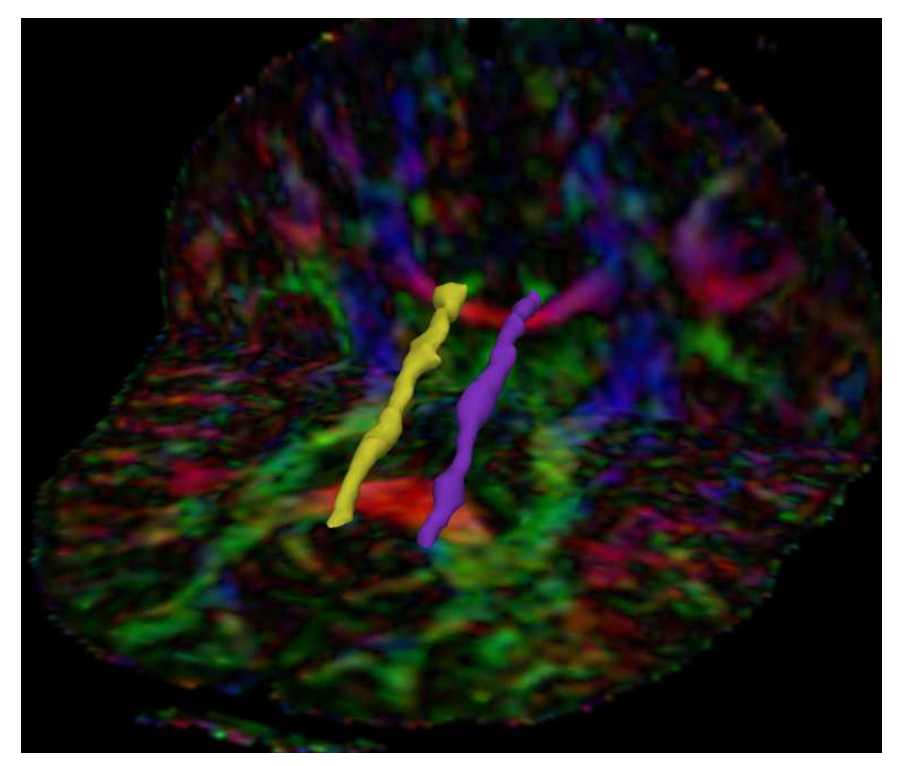

Figure 4: Result on DW-MRI data. The structures in yellow and magenta indicate the CB surface identified in the right and left hemisphere of the brain volume.

[4] E. Pichon. Novel methods for multidimensional image segmentation. PhD thesis, Georgia Institute of Technology, 2005.

[5] M. Rousson, T. Brox, R. Deriche, I. Projet Odyssee, and F. Sophia-Antipolis. Active unsupervised texture segmentation on a diffusion based feature space. Computer Vision and Pattern Recognition, 2003. Proceedings. 2003 IEEE Computer Society Conference on, 2, 2003.

[6] J.D. Schmahmann and D.N. Pandya. Fiber Pathways of the Brain. Oxford University Press, 2006.

[7] J.A. Sethian. Level set methods and fast marching methods. Cambridge University Press Cambridge, 1999.

[8] F. Wang, Z. Sun, L. Cui, X. Du, X. Wang, H. Zhang, Z. Cong, N. Hong, and D. Zhang. Anterior Cingulum Abnormalities in Male Patients With Schizophrenia Determined Through Diffusion Tensor Imaging, 2004.

[9] R.T. Whitaker. A Level-Set Approach to 3D Reconstruction from Range Data. International Journal of Computer Vision, 29(3):203-231, 1998. 